\title{
RBBP6 induces non-small cell lung cancer cell proliferation and high expression is associated with poor prognosis
}

\author{
QIU-SHI WANG $^{1,2}$, SHI-RONG WEI $^{1}$ and HUA-LIANG XIAO ${ }^{1}$ \\ Departments of ${ }^{1}$ Pathology and ${ }^{2}$ Clinical Biobank, Daping Hospital and Research Institute of Surgery, \\ Army Medical University, Chongqing 400042, P.R. China
}

Received April 8, 2019; Accepted January 7, 2020

DOI: $10.3892 / \mathrm{ol} .2020 .11403$

\begin{abstract}
Lung cancer is the most common cause of cancer-associated mortality in China with $85 \%$ of patients having non-small cell lung cancer (NSCLC). Identifying NSCLC driver genes and prognostic markers is critical to reducing these numbers. The studies of retinoblastoma binding protein 6 (RBBP6) performed on NSCLC is limited. The present study aimed to investigate the molecular function and the prognostic potential of RBBP6 in NSCLC using the A549 cell line and patient samples, respectively. The functional effect on cancer cell proliferation and prognostic value of RBBP6 were examined in vitro and in vivo using reverse transcription-quantitative PCR, immunofluorescence, immunohistochemistry (IHC) and xenograft implantation. The results demonstrated that RBBP6 mRNA expression was significantly higher in NSCLC tissues compared with in adjacent normal samples. When RBBP6 mRNA expression was interfered with using short hairpin RNA, A549 cell proliferation and xenograft tumor growth were reduced. Additionally, IHC and survival analysis demonstrated that patients with NSCLC with high expression levels of RBBP6 had a shorter median overall survival time compared with patients with low RBBP6 expression (31 vs. 51.5 months), and this was more prominent in stage I-II patients (43 vs. $>67$ months). High expression levels of RBBP6 indicated poor prognosis in patients with NSCLC. This may be due to the ability of RBBP6 to promote cancer cell proliferation. RBBP6 may be a potential prognostic biomarker and a therapeutic target for NSCLC.
\end{abstract}

Correspondence to: Dr Qiu-Shi Wang, Department of Clinical Biobank, Daping Hospital and Research Institute of Surgery, Army Medical University, 10 Changjiang Zhilu, Chongqing 400042, P.R. China

E-mail: wqsdpbl@163.com

Professor Hua-Liang Xiao, Department of Pathology, Daping Hospital and Research Institute of Surgery, Army Medical University, 10 Changjiang Zhilu, Chongqing 400042, P.R. China E-mail: dpbl_xhl@126.com

Key words: non-small cell lung cancer, retinoblastoma binding protein 6 , proliferation, prognosis

\section{Introduction}

Lung cancer is the most common cause of cancer-associated mortality in both men and women in China, with non-small cell lung cancer (NSCLC) accounting for nearly $85 \%$ of cases of mortality in 2015 (1). Although experimental and clinical studies $(2,3)$ have resulted in remarkable achievements, the prognosis for the majority of patients with NSCLC remains unsatisfactory, with a 5-year relative survival rate of $\sim 17 \%$ in 2016 (4). Therefore, more attention should be focused on finding novel and more reliable biomarkers for the prevention, diagnosis, treatment and prognosis of NSCLC.

The retinoblastoma binding protein 6 (RBBP6) gene, located on chromosome 16p11.2-p12, encodes a $200 \mathrm{kDa}$ protein with multiple repeat sequences. RBBP6 is a nuclear protein with a conserved $\mathrm{N}$-terminal ring-finger domain that is considered to be involved in protein degradation $(5,6)$. It has a p53 and retinoblastoma protein binding domain located near the C-terminus (7). Overexpression of RBBP6 leads to cell cycle arrest, a common feature of tumorigenesis (8), and is strongly associated with tumor progression in cervical and esophageal cancer (6). This suggests that RBBP6 may serve a critical role in the malignant phenotype of human cancer $(6,9,10)$. Motadi et al $(7)$ have demonstrated that RBBP6 mRNA and its protein products are expressed in human lung cancer. However, little is known regarding its clinical and pathological significance, particularly the prognostic value of RBBP6 expression in NSCLC.

The present study aimed to investigate the molecular function of RBBP6 in NSCLC cell proliferation by RNA silencing, and evaluated the association between RBBP6 expression and the prognosis of patients with NSCLC. The overall aim of the present study was to determine the value of RBBP6 as a potential prognostic marker for NSCLC.

\section{Materials and methods}

Patients and tissue samples. A total of 58 formalin-fixed and paraffin-embedded (FFPE) tissue samples (including 43 males and 15 females; mean age, 60.5 years; age range, $42-84$ years) and 23 pairs of fresh-frozen tissues (cancer and normal adjacent tissue, including 16 males and 7 females; mean age, 60.0 years; age range, $38-71$ years) of consecutive patients were obtained from the Department of Pathology and Clinical 
Biobank, respectively, of Daping Hospital (Chongqing, China) between March 2011 and December 2011. NSCLC was diagnosed by clinical pathologists. None of the patients received radiotherapy, chemotherapy or targeted therapy prior to surgery. All patients signed the consent to use their tissues and were informed that their clinical data may be published. The present study was approved by the Clinical Ethics Committee of Daping Hospital and Research Institute of Surgery, Army Medical University.

FFPE tissues from the 58 patients were used for pathological diagnosis according to the WHO pathological guideline (11). Among the FFPE tissues, 34 were adenocarcinoma, 18 were squamous carcinoma and 6 were not otherwise specified carcinoma (NOS). These tissues were used for immunohistochemistry (IHC). In addition, 23 pairs of fresh-frozen tissues were used to analyze RBBP6 mRNA expression. Clinical and pathological data (sex, age, location of cancer, smoking history, clinical stage and pathological diagnosis) of the patients were obtained from their medical records. Follow-up was performed by telephone every 3 months, starting from the date of primary diagnosis after the surgery and ending at the observable relapse (death).

Cell culture, transfection and xenograft in vivo tumor models. Short hairpin RNA (shRNA) bacterial clones of RBBP6 on the pLKO.1 vector were purchased from Sigma-Aldrich (Merck KGaA). The non-targeting scrambled sequence with the same vector (scrRNA-control) purchased from the Addgene, Inc. was used as the transfection control. The A549 cells were used as the blank control. The hairpin sequences of shRNA-RBBP6 and scrRNA were 5'-CCG GGATTGTCAGGAGGATTCCTATCTCGAGATAGGAAT CCTCCTGACAATCTTTTTG-3' and 5'-CCTAAGGTT AAGTCGCCCTCGCTCGAGCGAGGGCGACTTAACCTT AGG-3', respectively. The plasmid was extracted, amplified and then transduced into 293T cells. The lung cancer cell line A549 $\left(1 \times 10^{4}\right.$ cells/100 $\mu \mathrm{l} /$ well $)$ were cultured in DMEM supplemented with $1 \%$ penicillin-streptomycin and $10 \%$ FBS (Biological Industry) in a humidified atmosphere at $37^{\circ} \mathrm{C}$ with $5 \% \mathrm{CO}_{2}$ for $24 \mathrm{~h}$ prior to transfection. A549 cells were trypsinized and re-suspended in antibiotic-free media, and then mixed with shRNA RBBP6/Lipofectamine ${ }^{\mathrm{TM}} 2000$ transfection agent (Invitrogen, Thermo Fisher Scientific, Inc.) or scrRNA control/transfection agent complexes (100 nM). The cells were incubated in 24 -well plates at $37^{\circ} \mathrm{C}$ for $24 \mathrm{~h}$. Post-transfected cells were then harvested for subsequent analysis.

All procedures were performed in accordance with protocols approved by the Animal Welfare and Ethics Committee of the Army Medical University. Female BALB/C null mice $(n=9$; age, 5 weeks; weight, $18-20 \mathrm{~g}$ ) were provided by the Animal Center of Daping Hospital, Third Military Medical University (certificate no. scxk (Yu) 2002-0002; Chongqing, China). Mice were randomly divided into the control group and the shRNA-RBBP6 group ( $\mathrm{n}=3$ animals/group). They were subcutaneously injected with $5 \times 10^{6}$ transfected cells ( $5 \times 10^{6}$ non-transfected cells for mice in the control group) in $200 \mu 1$ PBS into the armpit of the left forelimb. All mice were kept in individual ventilated cages with food and water ad libitum at controlled temperature conditions $\left(22 \pm 1^{\circ} \mathrm{C}\right)$ with a
$12 \mathrm{~h} \mathrm{light/dark}$ cycle at 50\% humidity in the Animal Center of Daping Hospital. All animals were monitored once a week to identify body weight gain and tumor growth. A tumor was defined as a palpable mass recorded for $\geq 2$ consecutive weeks. At 3.5 weeks after grafting, when the tumors of the control group had grown to $1.0-1.5 \mathrm{~cm}$ in diameter, mice were sacrificed using cervical spine dislocation and the tumors were surgically removed. Tumor volume was calculated as (length $\mathrm{x}$ width $\left.{ }^{2}\right) / 2$.

RNA isolation, purification and reverse transcription-quantitative PCR (RT-qPCR). RBBP6 mRNA relative expression was analyzed using RT-qPCR and Cq methods (12). Fresh-frozen NSCLC cancer tissues and normal adjacent tissues $(>5 \mathrm{~cm}$ away from the cancer edge) were lysed using TissueLyser II (Qiagen $\mathrm{GmbH}$ ). Total RNA was harvested and purified using the RNeasy Mini kit (Qiagen GmbH). RNA quantity and quality were measured using the NanoDrop 2000 (Thermo Fisher Scientific, Inc.). Total RNA $(1 \mu \mathrm{g})$ was then reverse transcribed to cDNA using M-MLV Reverse Transcriptase (Thermo Fisher Scientific, Inc.) in a total volume of $20 \mu 1$. The reverse transcription was performed in 2 steps: $42^{\circ} \mathrm{C}$ for $60 \mathrm{~min}$ and $70^{\circ} \mathrm{C}$ for $10 \mathrm{~min}$ using $\mathrm{C} 1000$ Touch (Bio-Rad Laboratories, Inc.).

RBBP6 mRNA expression was determined by qPCR. Primer sequences were as follows: $R B B P 6$ forward, 5'-GTG TTTCCGTTGTGGTAAACCTGG-3' and reverse, 5'-CCA GTGTTGGTAAGCATTGCACC-3'; actin forward, 5'-TCA TGTTTGAGACCTTCAA-3' and reverse, 5'-GTCTTTGCG GATGTCCACG-3'. qPCR was performed using $10 \mu 12 \mathrm{X}$ SYBR Green Mastermix (Thermo Fisher Scientific, Inc.), $500 \mathrm{nM}$ primers, $2 \mu \mathrm{l}$ diluted cDNA (1:10) and distilled water to obtain a final volume of $20 \mu \mathrm{l}$. RT-qPCR was performed in a 2-step cycle using a LightCycler 480 II (Roche Diagnostics) as follows: $95^{\circ} \mathrm{C}$ for $10 \mathrm{~min} ; 40$ cycles of $95^{\circ} \mathrm{C}$ for $10 \mathrm{sec}$ and $60^{\circ} \mathrm{C}$ for $30 \mathrm{sec}$ (fluorescent signal measured at this step). The amplified sequence was short enough so an extension step was not used.

Western blot analysis. Cells were washed twice with cold PBS and then harvested $\left(\sim 3 \times 10^{6}\right.$ cells) by scraping the cell monolayers. Subsequently, the cells were lysed in $150 \mu 1$ RIPA buffer (Beyotime Institute of Biotechnology). Total protein was then separated from cell debris by centrifugation at $4^{\circ} \mathrm{C}$ at 14,000 x g for $10 \mathrm{~min}$, and quantified using the Pierce ${ }^{\circledast}$ BCA Protein Assay kit (Thermo Fisher Scientific, Inc.) Proteins were denatured at $95^{\circ} \mathrm{C}$ for $5 \mathrm{~min}$ and $30 \mu \mathrm{g} /$ well was loaded for electrophoretic separation in a $40 \%$ acrylamide gel at $80 \mathrm{~V}$ for $30 \mathrm{~min}$, followed by $100 \mathrm{~V}$ for $1 \mathrm{~h}$. Proteins were transferred onto a nitrocellulose membrane using the wet electro-transfer method (13) for $90 \mathrm{~min}$ at $100 \mathrm{~V}$ followed by blocking with $5 \%$ non-fat milk buffer at room temperature for $2 \mathrm{~h}$. The membrane was incubated with mouse monoclonal antibody RBBP6 (1:500; cat. no. M56; Santa Cruz Biotechnology, Inc.) and mouse monoclonal antibody actin (1:2,000; cat. no. C-2; Santa Cruz Biotechnology, Inc.) at $4^{\circ} \mathrm{C}$ overnight. Following incubation with horseradish peroxidase-linked goat and anti-mouse antibodies (1:5,000; cat. nos. ZDR-5117; OriGene Technologies Inc.) at room temperature for $60 \mathrm{~min}$. Immune-reactive bands were visualized using the Clarity 

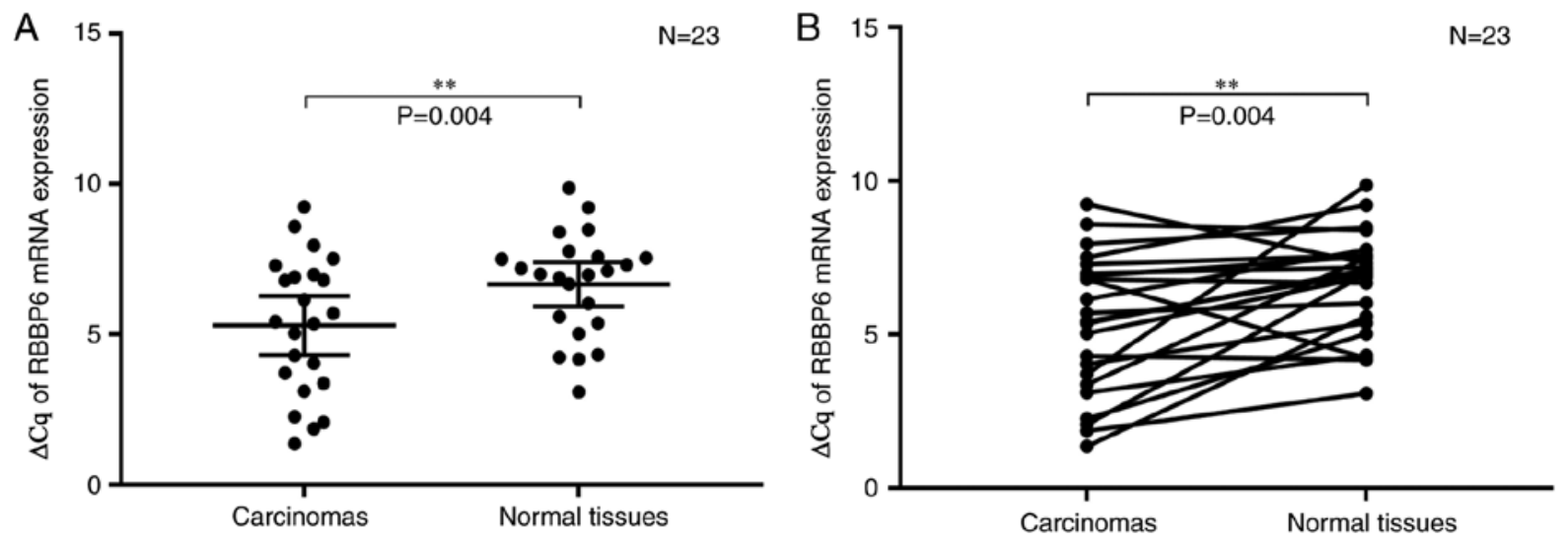

Figure 1. RBBP6 mRNA expression levels in 23 pairs of non-small cell lung cancer and normal adjacent tissues. (A) Mean RBBP6 expression (presented as $\Delta \mathrm{Cq}=\mathrm{Cq}_{\mathrm{RBBP}}-\mathrm{Cq}_{\mathrm{Actin}}$, where lower $\Delta \mathrm{Cq}$ indicates higher expression levels) in tumors was significantly higher than that in normal adjacent tissues ( $\left.\mathrm{P}=0.004\right)$. (B) Among the patients, 18/23 (78.3\%) exhibited high tumor RBBP6 expression compared with expression in normal adjacent tissues. ${ }^{* *} \mathrm{P}<0.01$. RBBP6, retinoblastoma binding protein 6 .

Western ECL Substrate kit (Bio-Rad Laboratories, Inc.). The blots were imaged using the FUSION FX5 system (Vilber Lourmat), and the densitometry was analyzed using the software ImageJ (version 1.8.0; National Institute of Health).

5-ethynyl-2'-deoxyuridine (EdU) assay. The EdU assay was used to evaluate A549 cell proliferation following shRNA inhibition of RBBP6 expression. A total of 5,000 cells were plated in $0.2 \mathrm{ml}$ of 96-well flat bottom plates with DMEM supplemented as mentioned above for 24, 48, 72 and $96 \mathrm{~h}$ following transfection, The control and transfected cells were harvested and incubated with $50 \mu \mathrm{M}$ EdU substrate in 96-well plates at $37^{\circ} \mathrm{C}$ for $2 \mathrm{~h}$. Following fixation with $4 \%$ paraformaldehyde at room temperature for $30 \mathrm{~min}$, cells were stained with EdU using the $1 \mathrm{X}$ Apollo ${ }^{\circledR}$ reaction kit (Guangzhou RiboBio Co., Ltd.) at room temperature for $30 \mathrm{~min}$ and reviewed using a fluorescent microscope (Leica Microsystems, Inc.).

IHC. Mouse monoclonal antibody RBBP6 (cat. no. M56; Santa Cruz Biotechnology, Inc.) was used for IHC analysis. Formalin-fixed and paraffin-embedded (4\% neutral formalin for $6-12 \mathrm{~h}$ at room temperature) tissues were sliced into $4-\mu \mathrm{m}$ thick sections. The tissues were subsequently deparaffinized and pretreated with $1 \mathrm{mmol} / \mathrm{lEDTA}$ at $\mathrm{pH} 9.0 \mathrm{in}$ a high-pressure cooker for $3 \mathrm{~min}$ and then treated with $3 \% \mathrm{H}_{2} \mathrm{O}_{2}$ for $10 \mathrm{~min}$. The slides were rehydrated in a descending alcohol series (100, 95 and $85 \%$ ). Subsequently, washing was performed for $3 \mathrm{~min}$ three times using $0.01 \mathrm{M}$ PBS at room temperature and the slides were incubated with an anti-RBBP6 antibody (1:200) in the humidified chamber at $4^{\circ} \mathrm{C}$ overnight. Next day, the slides were washed in 0.01 M PBS and then incubated with goat anti-mouse/rabbit IgG ready to use reagent (cat. no. V-6000; Origene Technologies, Inc.) at room temperature for $30 \mathrm{~min}$ and stained using 3,3'-diaminobenzidine for $5 \mathrm{~min}$ at room temperature. The slides were reviewed using a bright-field microscope (x100) (cat. no. BX41;Olympus Corp.) and Cell Sens Standard software (version 1.16; Olympus Corp.) blinded by two pathologists at the Department of Pathology, Army Medical University (Chongqing, China). The slides were scored for strong staining (3+), moderate staining (2+), faint staining (1+) and no staining (0) according to the method described (14). Tissues scored as $3+$ and $2+$ were classified as high expression, whereas $1+$ and $0+$ were defined as low expression.

Statistical analysis. Data was expressed as mean \pm SD and experiments were repeated three times. Two-tailed $\chi^{2}$ test and Fisher's exact test were used to assess RBBP6 mRNA expression levels between tumor tissues and normal adjacent tissues. The Kaplan-Meier method was used to assess the prognostic value of RBBP6 expression for patients with NSCLC. A log-rank test was used to compare survival curves and a Cox proportional hazards model was used to calculate univariate and multivariate hazard ratios for the variables. The one-way ANOVA with a post-hoc Tukey's test was used for multiple comparisons. $\mathrm{P}<0.05$ was considered to indicate a statistically significant difference. All analyses were performed using SPSS software (version 18.0; SPSS, Inc.).

\section{Results}

Increased RBBP6 mRNA expression levels in NSCLC tissues compared with in normal adjacent tissues. A total of 23 pairs of tumor and adjacent normal tissues from patients with NSCLC were used to assess RBBP6 mRNA expression levels. $\beta$-actin was used as the housekeeping gene. RBBP6 mRNA expression levels were represented as $\Delta \mathrm{Cq}\left(\Delta \mathrm{Cq}=\mathrm{Cq}_{\mathrm{RBBP}}-\mathrm{Cq}_{\text {Actin }}\right)$, where lower $\Delta \mathrm{Cqs}$ indicated higher expression. The mean expression levels of RBBP6 were significantly higher in tumors compared with in normal adjacent tissues $\left(\Delta \mathrm{Cq} 5.3_{\text {Tumor }}\right.$ vs. $6.67_{\text {Normal }}$, $\mathrm{P}=0.004$; Fig. 1A). Furthermore, the diagonals indicated that $18 / 23(78.3 \%$ ) patient tumor tissues had lower $\Delta \mathrm{Cq}$ (higher RBBP6 expression levels) compared with adjacent normal tissues, whereas only 5/23 (21.7\%) tumor tissues had higher $\Delta \mathrm{Cq}$ (lower RBBP6 expression levels) compared with the adjacent normal tissue (Fig. 1B).

RNA interference (RNAi)-mediated knockdown of RBBP6 reduces $A 549$ cell proliferation and xenograft tumor growth. shRNA-RBBP6 was transfected into the lung adenocarcinoma cell line A549. The RNAi knockdown efficiency was evaluated by examining mRNA expression levels and via western 
A

B
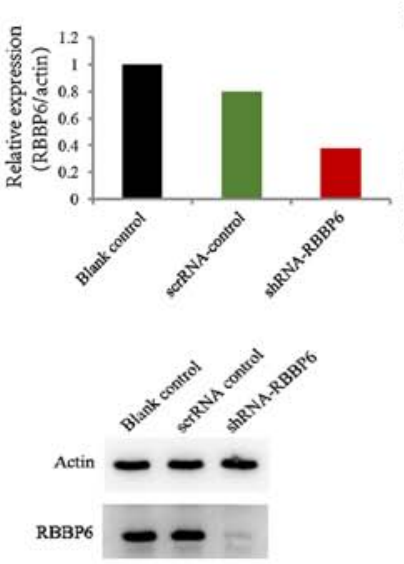

C

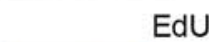

E

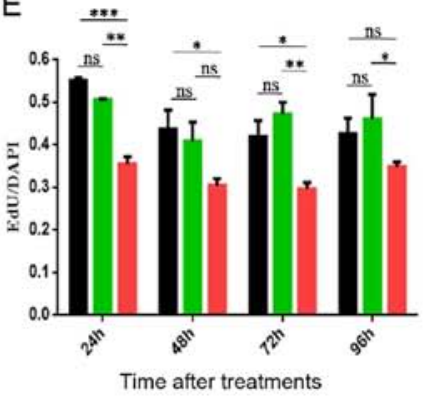

F

- Blank-control

-

$\mathrm{N}=3$
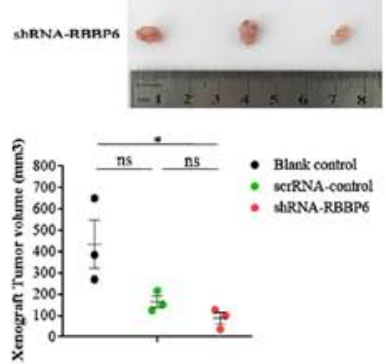

Overlay

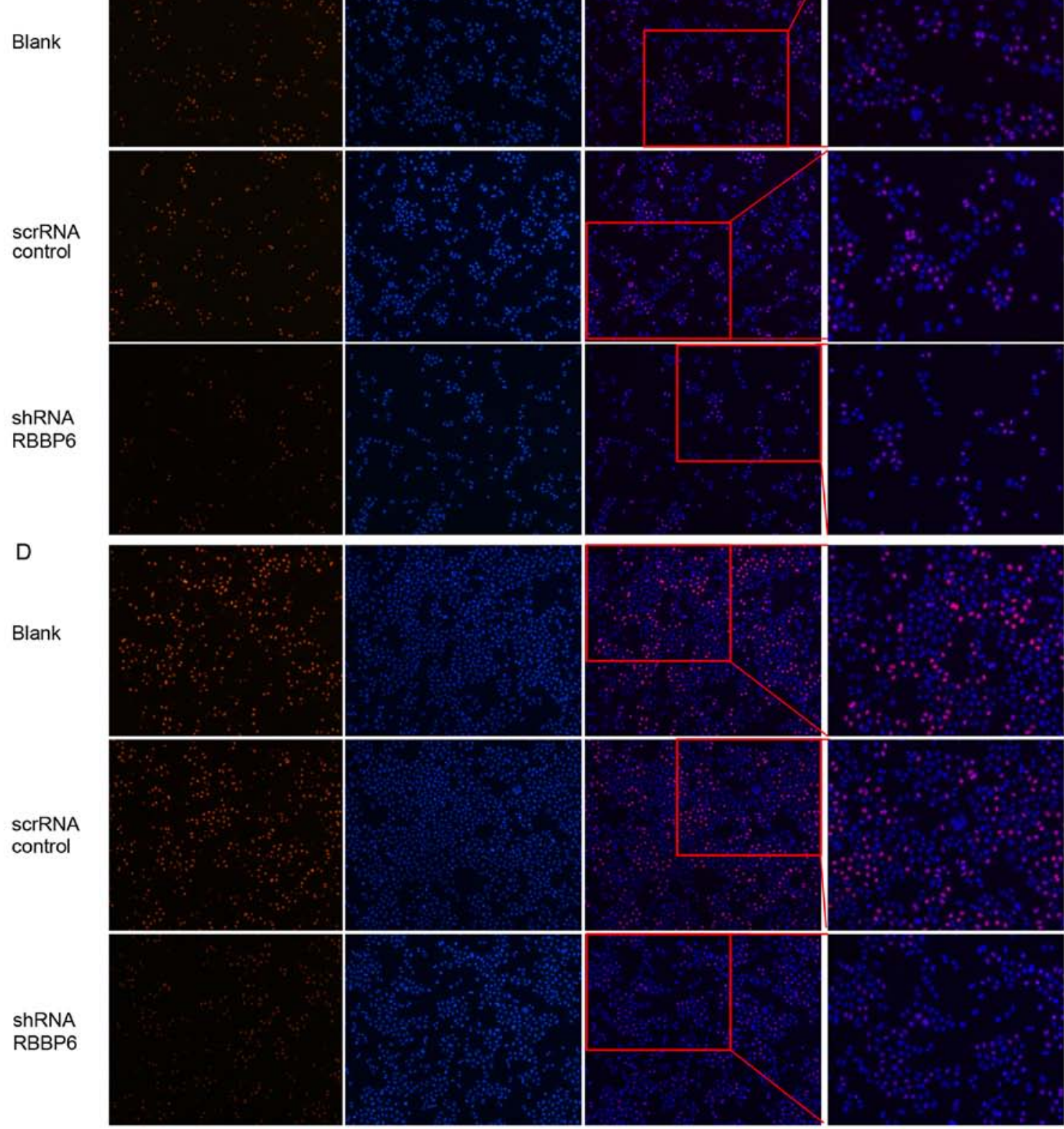

Figure 2. shRNA-RBBP6 reduces A549 cell proliferation and xenograft tumor growth. (A) Reverse transcription-quantitative PCR and (B) western blot analysis demonstrated effective transfection of shRNA-RBBP6 into A549 cells. EdU assays were performed 24, 48, 72 and $96 \mathrm{~h}$ post-transfection. Tumor cell proliferation presented by EdU assay is demonstrated at (C) 24 and (D) $96 \mathrm{~h}$ following transfection. Magnification, x100. Whereas after $96 \mathrm{~h}$, the cell proliferation of the shRNA-RBBP6 group recovered to some extent compared with (E) $72 \mathrm{~h}$. (F) In vivo assays demonstrated that shRNA-RBBP6 reduced xenograft tumor growth in BALB/C null mice. ${ }^{*} \mathrm{P}<0.05,{ }^{* * *} \mathrm{P}<0.01,{ }^{* * * *} \mathrm{P}<0.001$, as indicated. shRNA, short hairpin RNA; RBBP6, retinoblastoma binding protein 6; ns, not significant; scrRNA, scrambled RNA. 
Table I. Clinical characteristics of 58 patients with non-small cell lung cancer and RBBP6 protein expression.

\begin{tabular}{|c|c|c|c|}
\hline Characteristics & Patients $(n=58)(n=58)$ & RBBP6 IHC (-/+) (-/+) & RBBP6 IHC $(++/+++)(++/+++)$ \\
\hline \multicolumn{4}{|l|}{ Age, years } \\
\hline Mean & 60.5 & 57.2 & 64.5 \\
\hline \multicolumn{4}{|l|}{$\operatorname{Sex}, \mathrm{n}(\%)$} \\
\hline Male & $43(74.1)$ & 22 & 21 \\
\hline Female & $15(25.9)$ & 10 & 5 \\
\hline \multicolumn{4}{|l|}{ Smoking status, n (\%) } \\
\hline Never smoked & $24(41.4)$ & 14 & 10 \\
\hline Smokers & $34(58.6)$ & 18 & 16 \\
\hline \multicolumn{4}{|c|}{ Pathological classification, n (\%) } \\
\hline Adenocarcinoma & $34(58.6)$ & 20 & 14 \\
\hline Squamous carcinoma & $18(31.0)$ & 7 & 11 \\
\hline NOS & $6(10.4)$ & 5 & 1 \\
\hline \multicolumn{4}{|l|}{ Clinical stage, n (\%) } \\
\hline I-II & $43(74.1)$ & 21 & 22 \\
\hline III-IV & $15(25.9)$ & 11 & 4 \\
\hline
\end{tabular}

NOS, not otherwise specified; IHC, immunohistochemistry; RBBP6, retinoblastoma binding protein 6.

blot analysis (Fig. 2A and B). EdU assay results demonstrated that inhibition of RBBP6 expression reduced A549 cell proliferation at 24, 48, 72 and $96 \mathrm{~h}$ post-transfection compared with the blank and scrRNA control (Fig. 2C-E).

Female BALB/C null mice $(n=9)$ were divided into three groups, the blank control group $(n=3)$, the scrRNA control group $(n=3)$ and the shRNA-RBBP6 group $(n=3)$. Axillary lumps were observed at 2 weeks post subcutaneous injection of $5 \times 10^{6}$ cells. Compared with both the blank control group and the scrRNA control group, the shRNA-RBBP6 group exhibited a marked decrease in xenograft tumor volume at 3.5 weeks post-injection (Fig. 2F).

Higher RBBP6 expression in patients with NSCLC is associated with poor prognosis. IHC was used to analyze RBBP6 protein expression in 58 NSCLC FFPE samples, which comprised 34 adenocarcinoma, 18 squamous carcinoma and 6 NOS cases. Tumor samples were derived from 43 men $(74.1 \%)$ and 15 women (25.9\%), of which 34 were smokers (58.6\%), 43 were clinical stage I-II (74.1\%) and 15 were stage III-IV (25.9\%; Table I). The majority of RBBP6 was expressed in the nucleus with low expression in the cytoplasm (Fig. 3A). Among the tumor samples, 26/58 (44.8\%) had high RBBP6 expression and this was more frequently observed in males $(21 / 43 ; 48.8 \%)$ and smokers $(16 / 34 ; 47.1 \%)$ compared with females $(5 / 15 ; 33.3 \%)$ and non-smokers $(10 / 24 ; 41.7 \%)$, and less frequently observed in adenocarcinoma $(14 / 34 ; 41.2 \%)$ compared with squamous carcinoma $(11 / 18 ; 61 \%)$. However, the results were not statistically significant due to the small number of patients.

The statistical association between the median overall survival (mOS) of the 58 patients and RBBP6 expression was analyzed using the Kaplan-Meier method. Patients with low RBBP6 expression had longer mOS compared with patients with high RBBP6 expression (51.5 vs. 37 months; Fig. 3B), which indicated that higher RBBP6 expression was associated with poor prognosis. This result was not statistically significant. In order to compensate for the limited number of patients with stage III-IV cancer, the present study analyzed the prognostic value of RBBP6 expression for stage I-II patients. Patients with high RBBP6 expression had a mOS of 43 months, whereas patients with low RBBP6 expression had not reached the observable relapse at the indicated time interval (Fig. 3C). This result did not reach statistical significance.

\section{Discussion}

RBBP6, also known as p53-associated cellular protein testes derived (15), proliferation potential-related protein (16) or retinoblastoma binding Q protein 1 (17), serves an important role in tumorigenesis (9). In the present study, knockdown of RBBP6 expression by RNAi significantly reduced lung adenocarcinoma A549 cell proliferation and xenograft tumor growth. These results suggested that RBBP6 may promote tumorigenesis by increasing cancer cell proliferation. Furthermore, these results were consistent with previous studies which demonstrated that RBBP6 expression is significantly increased in esophageal tumors compared with normal adjacent tissues (6), and inhibition of RBBP6 expression by siRNAs significantly reduces breast cancer cell growth (18).

The present study demonstrated that RBBP6 induced cancer cell proliferation, and thus, may be an independent prognostic factor for NSCLC. Higher expression levels of RBBP6 in colon tumor tissues are associated with clinical stage, depth of tumor invasion, lymph node metastasis, distant metastasis and histologic grade (10). RBBP6 is highly expressed in cervical cancer tissues, particularly in patients with stage II or III cancer (19). The present study revealed similar prognostic value for RBBP6 in NSCLC. Patients with 
A
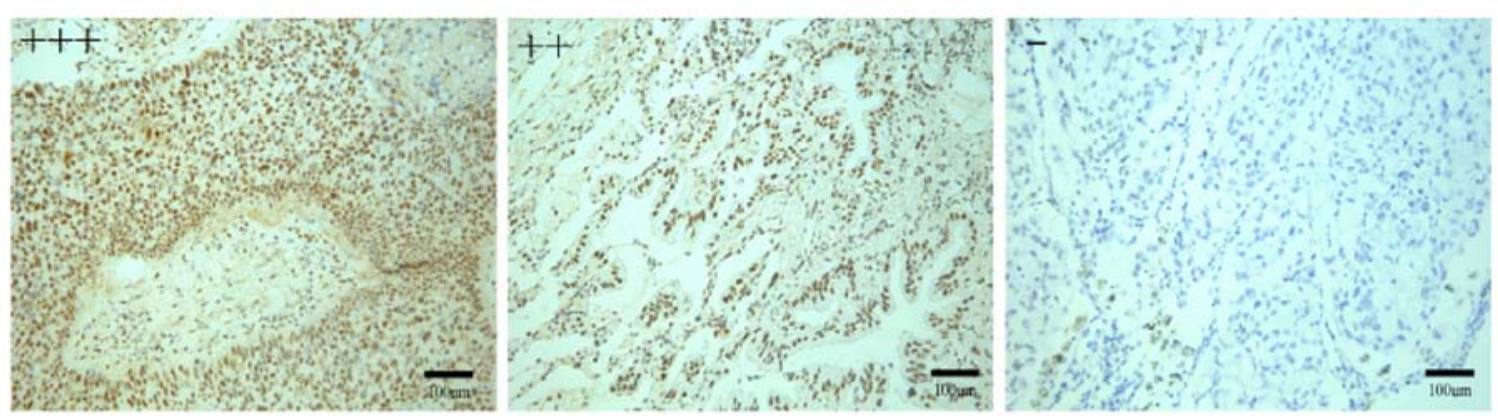

B
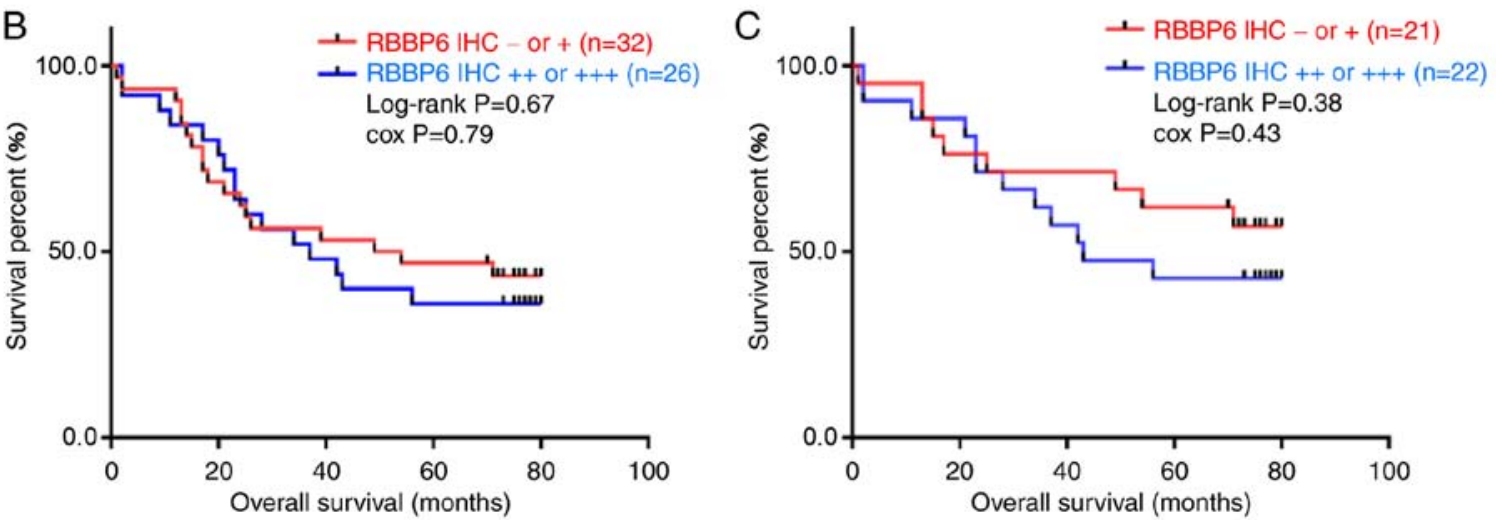

Figure 3. High expression of RBBP6 is associated with poor non-small cell lung cancer prognosis. (A) RBBP6 was mainly localized in the nucleus with minimal expression in the cytoplasm. Scale bar, $100 \mu \mathrm{m}$. (B) Among the 58 patients, patients with low RBBP6 expression had an improved mOS compared with patients with high RBBP6 expression. (C) Among the 43 patients with stage I-II, patients with low RBBP6 expression exhibited an improved mOS compared with patients with high RBBP6 expression. mOS, mean overall survival; IHC, immunohistochemistry; RBBP6, retinoblastoma binding protein 6.

high RBBP6 expression exhibited shorter mOS time compared with patients with low RBBP6 expression (31 vs. 51.5 months). However, the difference was not statistically significant. Due to the limited number of patients with stage III-IV cancer, the present study analyzed the median overall survival for the 43 patients with stage I and II cancer. Similar results were observed, indicating that higher RBBP6 expression group was associated with short term OS (43 vs. $>67$ months) compared with the lower RBBP6 expression group. Similarly, the difference was not statistically significant, this might be due to the small number of involved cases. Motadi et al (7) have reported that RBBP6 is highly expressed in human lung cancer, but, to the best of our knowledge, its prognostic value has not been evaluated previously.

The functional effect of RBBP6 on promoting NSCLC cancer cell proliferation may depend on its interaction with anti-oncogenes P53 or Rb. RBBP6 may be involved in the degradation of $\mathrm{p} 53$, thereby enhancing cell proliferation in lung cancer (7). In addition, the ability of RBBP6 to bind and interact with Rb1 may function in cell terminal differentiation (16). Therefore, RBBP6 may be involved in the regulation of the cell cycle, apoptosis and cell differentiation $(8,19-21)$.

In summary, the present study demonstrated that knockdown of RBBP6 reduced the proliferation of NSCLC cells. Patients with NSCLC with high RBBP6 expression had shorter overall survival compared with patients with low RBBP6 expression. RBBP6 may be a potential prognostic biomarker and a therapeutic target for NSCLC. However, the findings of the present study need to be validated in larger patient cohorts.

\section{Acknowledgements}

The authors would like to thank Professor Mengxia Li (Cancer Center, Daping Hospital and Research Institute of Surgery, Army Medical University, Chongqing, China) for providing the scrRNA control and A549 cell line.

\section{Funding}

The present study was supported by The Frontier and Applied Basic Scientific Research Project of Chongqing (grant no. cstc2015jcyjA10058 to QSW).

\section{Availability of data and materials}

The datasets used and/or analyzed during the present study are available from the corresponding author on reasonable request.

\section{Authors' contributions}

QSW and HLX conceived the study. SRW performed most experiments and raw data analysis. SRW contributed to experiment preparation and cell culture. All authors read and approved the final manuscript.

\section{Ethics approval and consent to participate}

The present study was approved by the Clinical Ethics Committee of Daping Hospital and Research Institute of 
Surgery, Army Medical University (Chongqing, China). Patients provided informed written consent and were informed that their clinical data may be used in publications.

\section{Patient consent for publication}

Not applicable.

\section{Competing interests}

The authors declare that they have no competing interests.

\section{References}

1. Chen W, Zheng R, Baade PD, Zhang S, Zeng H, Bray F, Jemal A Yu XQ and He J: Cancer statistics in China, 2015. CA Cancer J Clin 66: 115-132, 2016.

2. Herbst RS, Morgensztern D and Boshoff C: The biology and management of non-small cell lung cancer. Nature 553: 446-454, 2018.

3. Schiller JH: A new standard of care for advanced lung cancer. N Engl J Med 378: 2135-2137, 2018.

4. Miller KD, Siegel RL, Lin CC, Mariotto AB, Kramer JL, Rowland JH, Stein KD, Alteri R and Jemal A: Cancer treatment and survivorship statistics, 2016. CA Cancer J Clin 66: 271-289, 2016.

5. Pugh DJ, Ab E, Faro A, Lutya PT, Hoffmann E and Rees DJ: DWNN, a novel ubiquitin-like domain, implicates RBBP6 in mRNA processing and ubiquitin-like pathways. BMC Struct Biol 6: 1, 2006

6. Yoshitake Y, Nakatsura T, Monji M, Senju S, Matsuyoshi H, Tsukamoto H, Hosaka S, Komori H, Fukuma D, Ikuta Y, et al: Proliferation potential-related protein, an ideal esophageal cancer antigen for immunotherapy, identified using complementary DNA microarray analysis. Clin Cancer Res 10: 6437-6448, 2004

7. Motadi LR, Bhoola KD and Dlamini Z: Expression and function of retinoblastoma binding protein 6 (RBBP6) in human lung cancer. Immunobiology 216: 1065-1073, 2011.

8. Mbita Z, Meyer M, Skepu A, Hosie M, Rees J and Dlamini Z: De-regulation of the RBBP6 isoform 3/DWNN in human cancers. Mol Cell Biochem 362: 249-262, 2012.

9. Chibi M, Meyer M, Skepu A, G Rees DJ, Moolman-Smook JC and Pugh DJ: RBBP6 interacts with multifunctional protein YB-1 through its RING finger domain, leading to ubiquitination and proteosomal degradation of YB-1. J Mol Biol 384: 908-916, 2008.

10. Chen J, Tang H, Wu Z, Zhou C, Jiang T, Xue Y, Huang G, Yan D and Peng Z: Overexpression of RBBP6, alone or combined with mutant TP53, is predictive of poor prognosis in colon cancer. PLoS One 8: e66524, 2013.
11. Travis WD, Bramblilla E, Müller-Hermelink HK and Harris CC (eds): Tumours of the Lung, Pleura, Thymus and Heart. In: Pathology and Genetics. World Health Organization (WHO), IARC Press, Lyon, 2004.

12. Livak KJ and Schmittgen TD: Analysis of relative gene expression data using real-time quantitative PCR and the 2(-Delta Delta C(T)) method. Methods 25: 402-408, 2001.

13. Yao Z, Fenoglio S, Gao DC, Camiolo M, Stiles B, Lindsted T, Schlederer M, Johns C, Altorki N, Mittal V, et al: TGF-beta IL-6 axis mediates selective and adaptive mechanisms of resistance to molecular targeted therapy in lung cancer. Proc Natl Acad Sci USA 107: 15535-15540, 2010.

14. Li L, Luo Q, Xie Z, Li G, Mao C, Liu Y, Wen X, Yin N, Cao J, Wang J, et al: Characterization of the expression of the RNA binding protein eIF4G1 and its clinicopathological correlation with serous ovarian cancer. PLoS One 11: e0163447, 2016.

15. Simons A, Melamed-Bessudo C, Wolkowicz R, Sperling J, Sperling R, Eisenbach L and Rotter V: PACT: Cloning and characterization of a cellular p53 binding protein that interacts with Rb. Oncogene 14: 145-155, 1997.

16. Witte MM and Scott RE: The proliferation potential protein-related (P2P-R) gene with domains encoding heterogeneous nuclear ribonucleoprotein association and $\mathrm{Rb} 1$ binding shows repressed expression during terminal differentiation. Proc Natl Acad Sci USA 94: 1212-1217, 1997.

17. Sakai Y, Saijo M, Coelho K, Kishino T, Niikawa N and Taya Y: cDNA sequence and chromosomal localization of a novel human protein, RBQ-1 (RBBP6), that binds to the retinoblastoma gene product. Genomics 30: 98-101, 1995.

18. Moela P, Choene MM and Motadi LR: Silencing RBBP6 (Retinoblastoma Binding Protein 6) sensitises breast cancer cells MCF7 to staurosporine and camptothecin-induced cell death. Immunobiology 219: 593-601, 2014.

19. Moela P and Motadi LR: RBBP6: A potential biomarker of apoptosis induction in human cervical cancer cell lines. Onco Targets Ther 9: 4721-4735, 2016.

20. Scott RE, Giannakouros T, Gao S and Peidis P: Functional potential of P2P-R: A role in the cell cycle and cell differentiation related to its interactions with proteins that bind to matrix associated regions of DNA? J Cell Biochem 90: 6-12, 2003.

21. Dlamini Z, Rupnarain C, Naicker S, Hull R and Mbita Z: Expression analysis and association of RBBP6 with apoptosis in colon cancers. J Mol Histol 47: 169-182, 2016.

(i) $(-)$ This work is licensed under a Creative Commons Attribution-NonCommercial-NoDerivatives 4.0 International (CC BY-NC-ND 4.0) License. 